\title{
Possible Detection of a Pair Instability Supernova in the Modern Universe, and Implications for the First Stars
}

\author{
Nathan Smith \\ Astronomy Department, University of California, Berkeley
}

\begin{abstract}
.
SN 2006gy radiated far more energy in visual light than any other supernova so far, and potential explanations for its energy demands have implications for galactic chemical evolution and the deaths of the first stars. It remained bright for over 200 days, longer than any normal supernova, and it radiated more than $10^{51}$ ergs of luminous energy at visual wavelengths. I argue that this Type IIn supernova was probably the explosion of an extremely massive star like Eta Carinae that retained its hydrogen envelope when it exploded, having suffered relatively little mass loss during its lifetime. That this occurred at roughly Solar metallicity challenges current paradigms for mass loss in massive-star evolution. I explore a few potential explanations for SN2006gy's power source, involving either circumstellar interaction, or instead, the decay of ${ }^{56} \mathrm{Ni}$ to ${ }^{56} \mathrm{Co}$ to ${ }^{56} \mathrm{Fe}$. If SN $2006 \mathrm{gy}$ was powered by the conversion of shock energy into light, then the conditions must be truly extraordinary and traditional interaction models don't work. If SN 2006gy was powered by radioactive decay, then the uncomfortably huge ${ }^{56} \mathrm{Ni}$ mass requires that the star exploded as a pair instability supernova. The mere possibility of this makes SN $2006 \mathrm{gy}$ interesting, especially at this meeting, because it is the first good candidate for a genuine pair instability supernova.
\end{abstract}

Keywords: supernovae, SN 2006gy

PACS: 26.30.-k, 97.10.Me, 97.20.Pm, 97.20.Wt, 97.30.Eh, 97.30.Sw, 97.60.Bw, 98.38.Mj

\section{INTRODUCTION}

For the purposes of this meeting, the main relevant point is to ask whether or not SN 2006gy was really a pair instability supernova (PISN). I'll get into that later, but the short version is that we are not really sure. Its basic observed properties (high luminosity, long duration, and slow expansion speed of a heavy $\mathrm{H}$ envelope) seem consistent with the basic attributes of a PISN. On the other hand, alternative scenarios - while somewhat problematic and perhaps equally extraordinary - are hard to rule out conclusively. Nevertheless, it is worth discussing SN 2006gy in the context of PISNe, because if it was one, it may have some far-reaching implications for the pollution of interstellar matter and for learning about the deaths of the first stars. First, though, I'll just list some basic observables of SN 2006gy taken from Smith et al. (2007) and Ofek et al. (2007):

- The host galaxy was NGC 1260, which is a peculiar S0/Sa galaxy with sufficient evidence for star formation to make the presence of very massive stars plausible. SN 2006gy was about 300 pc from the galaxy's nucleus, and may have been near a spiral arm. The distance to NGC 1260 is $73.1 \mathrm{Mpc}$.

- The average metallicity of the host galaxy is roughly Solar $\left(\mathrm{Z} \simeq 0.63 \mathrm{Z}_{\odot}\right)$. We do not have a very good estimate of the metallicity at SN 2006gy's specific position in the galaxy.

- The peak bolometric luminosity was at least $\sim 5 \times 10^{10} \mathrm{~L}_{\odot}\left(M_{R} \simeq-22\right)$, and the total radiated energy in visible light during the first $\sim 200$ days was at least $1.4 \times 10^{51}$ ergs. This is 10 to 100 times more luminous than typical Type Ia and core collapse supernovae, respectively, and more than a factor of 100 greater than the total radiated energy of most SNe.

- In addition to being bright at its peak, the light curve of SN 2006gy evolved very slowly, taking more than 70 days to reach that peak, and even longer to decline (Fig. 1). This is unlike other SNe.

- Spectroscopically, SN 2006gy was a Type IIn supernova, meaning that it had narrow lines of hydrogen. This indicates that the star retained much of its original $\mathrm{H}$ envelope until the time it died.

- The $\mathrm{H} \alpha$ profile is actually rather complicated (Fig. 2), showing a very narrow P Cygni feature from slow-moving circumstellar material at $130-260 \mathrm{~km}$ $\mathrm{s}^{-1}$, plus a broader emission component of a few thousand $\mathrm{km} \mathrm{s}^{-1}$ (still much narrower than most supernovae). The speed of a few hundred $\mathrm{km} \mathrm{s}^{-1}$ indicated by the narrow component is too fast for a red supergiant (RSG) wind, but is consistent with the wind of a luminous blue variable (LBV) like $\eta$ Carinae.

- The expansion speed indicated by broad P Cygni absorption in the $\mathrm{H} \alpha$ line was about $4,000 \mathrm{~km} \mathrm{~s}^{-1}$, which did not change perceptably in the first $\sim 200$ days. In other words, the SN blast wave did not 


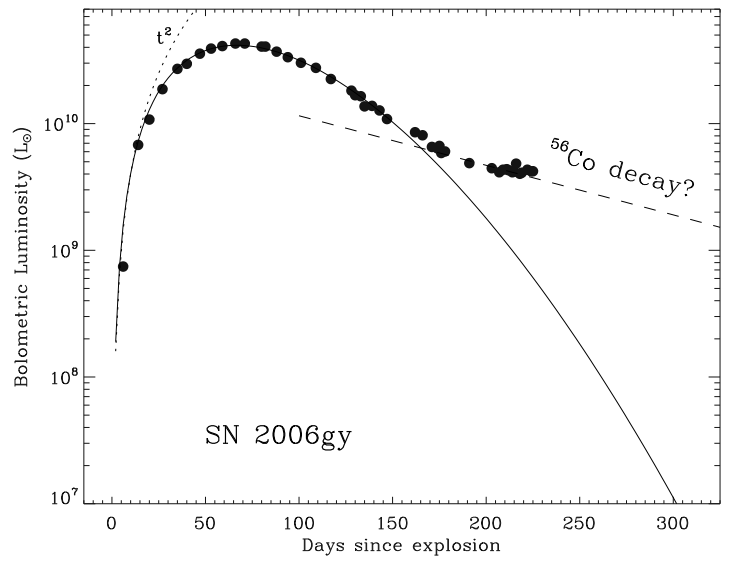

FIGURE 1. An approximation of the bolometric light curve of SN 2006gy adopting the unfiltered red magnitude with no bolometric correction (see Smith et al. 2007). It may therefore be an underestimate of the true luminosity. Although it uses the KAIT lightcurve from Smith et al. (2007), this figure is actually from Smith \& McCray (2008), using a simple thermal diffusion model to approximate the main part of the light curve, followed by a dashed line representave of radioactive decay from about $8 \mathrm{M}_{\odot}$ of $\mathrm{Ni}$.

decelerate much during a time when the SN emitted more than $10^{51}$ ergs of light. That will be important later.

- SN 2006gy was detected in soft X-rays by Chan$d r a$, and was spatially resolved from the nucleus of the galaxy, which appears to be an active nucleus. Analysis of the X-rays detected near the time of peak visual light of SN 2006gy implies a mass-loss rate for the progenitor's wind of no more than about $5 \times 10^{-4} \mathrm{M}_{\odot} \mathrm{yr}^{-1}$. The few X-rays that were detected (only 4 ! ...but a $10 \sigma$ detection) were all soft X-rays.

\section{SN 2006gy AS A PISN?}

The idea of PISNe has been around for four decades (Barkat, Rakavy, \& Sack 1967; Bond, Arnett, \& Carr 1984; Heger \& Woosley 2002), but we have yet to observationally verify that such explosions exist. While the case is still indefinite, SN 2006gy provides us with the first good candidate that matches the basic expected properties of a PISN. Namely, as described by Smith et al. (2007), it fits qualitative predictions for model lightcurves of PISNe (e.g., Scannapieco et al. 2005) in that it was extremely luminous, it had a slow rise time and long duration, it had slow expansion speeds because of the heavy envelope, and it was hydrogen rich. While

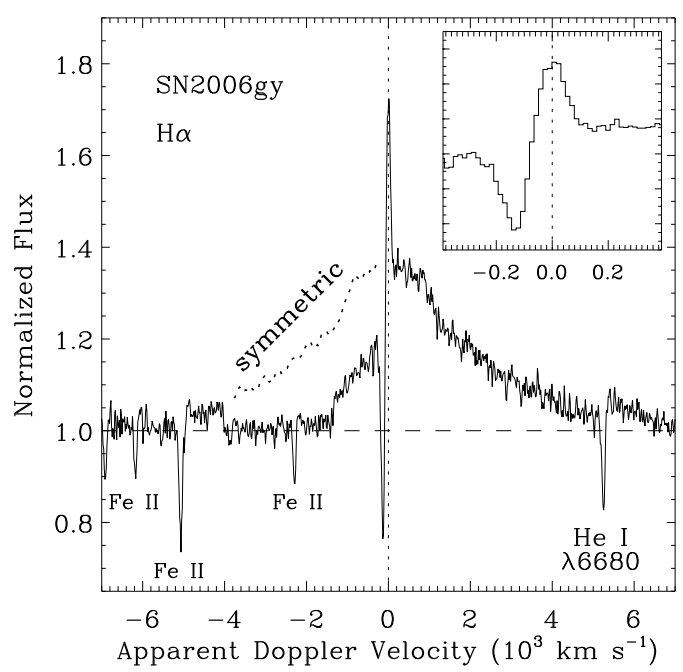

FIGURE 2. The $\mathrm{H} \alpha$ profile of SN 2006gy near the time its lightcurve peaked (from Smith et al. 2007). The inset shows an expanded version of the narrow P Cygni feature from the CSM. The dotted line is a reflection of the red side of the line, showing how a symmetric profile compares to the observed line shape.

the light curve of SN 2006gy does not precisely fit the predictions of Scannapieco et al., we should remember that those models were for zero metallicity stars with no mass loss, since that was the place PISNe were generally expected to occur. Direct application of those models to massive stars in the modern Universe at roughly Solar metallicity is probably unwise. While the progenitor of SN 2006gy did retain much of its original H enelope, it clearly has also suffered substantial mass loss prior to explosion, evidenced by emission from dense circumstellar material. Including some mass loss (and changing the mass of material through which radiation must diffuse) can drastically alter the shape of the light curve that one calculates. Some more recent work on PISN models described at this meeting in talks by K. Nomoto, D. Kasen, and T. Young shows different predictions for the light curves from different assumptions.

So far, the PISN model seems to be a viable explanation for SN 2006gy. A critical test will be its future behavior; SN 2006gy is currently behind the Sun and is unobservable. If its late-time decline reflects ${ }^{56} \mathrm{Co}$ decay, then this can be proof positive that SN 2006gy was a PISN. We should remember, though, that there may be some trickery involved. For example, the visual/red light curve in Figure 1 is not really a bolometric lightcurve. As the SN expands and cools, its bolometric flux may shift to the red and infrared, so a careful assessment of the multiwavelength properties need to be considered before we can say that it has faded faster than the radioactive decay rate (in fact, this may be part of the explanation 
for the leveling-off of the light curve at late times in Fig. 1). Also, late-time photometry will be tricky because of the background galaxy light, and then there's always the possibility of obscuration from dust formation. Thus, we should expect that sensitive IR observations will be critical to the nature of SN 2006gy in the immediate future. Another approach to evaluate our confidence in the viability of the PISN is to examine possible alternative explanations, as described next.

\section{ALTERNATIVES: CONVERSION OF SHOCK ENERGY INTO LIGHT}

Efficient conversion of blast wave kinetic energy into visual light is the only potentially-viable alternative to radioactive decay as a power source for the tremendous luminosity and total radiated energy of SN 2006gy. This is the current best-bet explanation for the luminosity and spectral properties of the class of Type IIn supernovae (e.g., ref), which can be more luminous than normal Type II-P supernovae, and show evidence for dense circumstellar material (CSM) in their spectra. The spectrum of SN 2006gy showed some clear characteristics in common with the Type IIn class (Smith et al. 2007; Ofek et al. 2007), including its relatively narrow $\mathrm{H} \alpha$ line. Aspects of the CSM interaction hypothesis for SN 2006gy have been discussed in detail by Ofek et al. (2007), Smith et al. (2007), Woosley et al. (2008), and Smith \& McCray (2008). The main consideration for the CSM-interaction hypothesis is that because of the extraordinarily-high luminosity of SN 2006gy, the CSM must be very dense and massive. I'll return to this momentarily.

The basic idea of how an interaction model can generate the extra high luminosity is that the SN blast wave expands out into a dense CSM. The CSM is swept up into a dense post-shock cooling layer that emits the observed visual continuum luminosity. In that scenario, the high luminosity phase can last until the shock runs past the densest CSM, or until the shock runs out of energy (you might call these cases "density bounded" or "energy bounded", reminiscent of terms for H II regions).

Ofek et al. (2007) first suggested this type of CSMinteraction model for SN 2006gy, discussed primarily in the framework of a Type Ia supernova interacting into a dense H-rich CSM. This is similar to what had been proposed for the recent luminous SNe 2002ic and 2006gj (Hamuy et al. 2003; Aldering et al. 2006). Smith et al. (2007) and Woosley et al. (2008) favored a somewhat different interpretation that SN 2006gy had been a very massive star that created its dense CSM when it suffered a giant outburst analogous to that of $\eta$ Carinae, occurring in the decade immediately before the SN. Observationally, such an outburst would probably have appeared similar to LBVs. The cause of LBV-type outbursts is not known, but one potential explanation is that it may have been triggered by the pulsational pair instability of Heger \& Woosley (2002). Even with an explanation for how to create the dense CSM, however, the case of SN 2006gy presents further constraints that complicate the normal Type IIn or CSM-interaction interpretation..

Because of the extreme energy demands of SN 2006gy, there are a couple things to keep in mind as we are considering a CSM-interaction model that we don't usually need to worry about in fainter Type IIn supernovae. First, since the total radiated energy is more than about $10^{51}$ ergs (Smith et al. 2007), the very efficient conversion of kinetic energy to light would drain a canonical SN blast wave of essentially all its available energy. Thus, for a normal SN, the expansion speed deduced from the spectrum should decelerate and eventually slow to a crawl as the energy is released as light. This did not happen in SN 2006gy, which showed a relatively constant expansion speed of about $4000 \mathrm{~km}$ $\mathrm{s}^{-1}$ throughout the time when it emitted its tremendous $10^{51}$ ergs of visual light (Smith et al. 2007). This requires either that some other source powers the visual light (like ${ }^{56} \mathrm{Co}$ decay), or that the available reservoir of $\mathrm{KE}$ was much more than $10^{51}$ ergs. Either case is exotic.

Second, even if we allow high efficiency in converting $\mathrm{KE}$ into light and we allow for very energetic explosion, we still run into other fundamental obstacles having to do with radiative transfer. In order to convert more than $10^{51}$ ergs of KE into light, a great deal of mass is required in the CSM - roughly $10 \mathrm{M}_{\odot}$ or more (Smith et al. 2007; Ofek et al. 2007) — ejected in the decade or so just before the SN. The fundamental problem, as pointed out recently by Smith \& McCray (2008), is that this amount of mass in the CSM would make that same CSM very opaque, so that the radiation could not escape even if it could be generated! This makes it impossible for conventional CSM-interaction models to explain SN 2006gy, because they rely on the continuous generation of visual light as the blast wave sweeps through the CSM.

Smith \& McCray (2008) suggest a possible way to circumnavigate this paradox that may rescue the CSMinteractionhypothesis for SN 2006gy. It may still draw its power from the conversion of KE into light, but in a way that is different from the continous CSM interaction models suggested by Ofek et al. (2007) and Woosley et al. (2007). Namely, Smith \& McCray proposed that the visual luminosity results from the diffusion of shockdeposited thermal energy escaping from an extended circumstellar envelope after it has already been overrun and accelerated by the blast wave. This is analogous to the Type II models of SNe in extended red supergiant envelopes (Falk \& Arnett 1973), except that here the envelope is not bound to the star, having been ejected in an LBV-like event a few years earlier. The $\sim 10 \mathrm{M}_{\odot} \mathrm{LBV}$ 
envelope was initially very opaque ( $\tau \simeq 300)$ at the time the $\mathrm{SN}$ occurred, and the delayed escape of radiation then occurs as photons diffuse out of the shocked envelope as it expands and thins. See Smith \& McCray (2008) for further details.

Now, SN 2006gy did have a spectrum that resembled Type IIn supernovae, as noted above, suggesting ongoing CSM-interaction as well. How can that be if the shock interaction with the shell was opaque? Well, after the blast wave breaks free of the opaque shell (which is when the visual light curve begins to rise) it will continue to expand into whatever CSM happens to reside there which we might expect to be a normal (i.e. non-outburst) stellar wind. In fact, the mass-loss rate derived from the usual interaction diagnostics like $\mathrm{H} \alpha$ and the soft $\mathrm{X}$-rays detected by Chandra indicated a fairly modest mass-loss rate that appeared entirely consistent with a normal stellar wind from a luminous blue supergiant star (Smith et al. (2007). That lower mass-loss rate would be nowhere near enough to account for the continuum luminosity of SN 2006gy, but it could give rise to the characteristic Type IIn spectrum.

The continuum luminosity needs a different source. Smith et al. (2007) noted several additional difficulties with a conventional CSM interaction model that could be explained by a PISN if the visual luminosity arises in the ejecta and not the ongoing interaction region In the photon diffusion model of Smith \& McCray (2008), the emitting geometry is similar to the situation where the bulk of the continuum luminosity arises in central stellar ejecta and not the ongoing CSM interaction region, so this model may be able to satisfy the obserational constraints as well as the PISN hypothesis. Either one is still viable at this point, but late-time observations may soon be able to give us an answer.

\section{MASS LOSS IN THE EVOLUTION OF MASSIVE STARS}

Whatever interpretation correctly explains the high luminosity of SN 2006gy, it is clear that the extreme energy demands require the death of a very mass star that may have started its life near the upper mass limit for stars. The facts that this star died with much of its $\mathrm{H}$ envelope intact and that it apparently had a mysterious outburst just before its final death as a SN are not understood in our current understanding of stellar evolution. A very massive star dying without shedding much of its mass also smells a little bit like the first stars, but this one happened at roughly Solar metallicity.

How can this be? Recent years have seen a revision in our thoughts about mass loss in the evolution of massive stars. One important clue is that several lines of evidence point to the fact that winds of hot stars may be highly clumped. This, in turn, means that mass-loss rates derived from density-squared diagnostics like $\mathrm{H} \alpha$ and radio continuum emission have severely overestimated mass loss rates. This conclusion derived from years of work done by many groups; a summary and relevant references can be found in Smith \& Owocki (2006). The main conclusion (Smith \& Owocki 2006) is that metallicitydependent line-driven winds of $\mathrm{O}$ stars do not shed very much mass in a star's lifetime, and therefore cannot account for the stripping of $\mathrm{H}$ envelopes that harkens the formation of Wolf-Rayet (WR) stars.

Nevertheless, WR stars do exist so some massive stars must be able to shed their $\mathrm{H}$ envelopes by some other means. Smith \& Owocki pointed out that the best candidates for the mass-loss mechanism that may make up the difference are the continuum-driven eruptions of LBVs, where a star can shed $10 \mathrm{M}_{\odot}$ or more in a single event that lasts less than a decade. These event are observed, but we still do not understand what triggers them.

On the other hand, the existence of Type IIn SNe like SN 2006gy and others indicates that some massive stars evolve differently - they do not fully shed their $\mathrm{H}$ envelopes, even through LBV eruptions, and die before they can become a WR star. In that case, a major goal in massive star evolution will be to determine how important these episodic outbursts are at different values of metallicity, initial mass, initial rotation rates, etc., and reevaluating the fates of massive stars as these quantities vary.

\section{IMPLICATIONS FOR THE FIRST STARS}

A subtle implication of SN 2006gy is that in some respects, and in some special cases, the evolution and deaths of very massive stars in the modern Universe might not be so terribly different from the first stars after all - at least in terms of their mass loss properties. If SN 2006gy was a PISN, it obviously has potential to inform our views about the deaths of the first stars and our interpretations of the role of PISNe in galactic chemical evolution. Even if SN 2006gy turns out not to have been a PISN, however, the fact remains that it reached the end of its life and exploded without losing much of its initial mass. This may suggest that studying massive stars in the local Universe may still tell us some things about the evolution of the first stars. Namely, with much lower mass-loss rates, the mass-loss evolution and angular momentum evolution of modern stars may, in some cases, be similar to early stars.

By the same token, very massive stars in the local Universe may then offer some sobering words of caution about our understanding of the first stars. For example, in recent years it has turned out that many of our as- 
sumptions about mass loss and the evolution of massive stars at Solar metallicity has turned out to be wrong. It seems to be the case that the most massive stars do not shed most of their mass in metallicity-dependent linedriven winds, and can instead shed much more mass in continuum-driven outbursts like the outbursts of LBVs (see Smith \& Owocki 2006). This is for stars in the local Universe where we have been collecting observational data for many decades. What does this imply about our understanding of the first stars, for which it will be some time before we have any direct observational tests?

Finally, the simple fact tha SN 2006gy was so bright suggests that there is some fraction of $\mathrm{SNe}$ - perhaps a small fraction - that can potentially be detected to much greater distances than conventional SNe. If SN 2006gy was indeed a PISN, then this obviously bodes well for our hopes of directly detecting the first SNe with future instruments like JWST. Again, though, even if SN 2006y was not a true PISN, there may be hope of detecting other SNe like it far back in time. If it wasn't a PISN, then the reason SN 2006gy was so bright was because it suffered a giant LBV-like mass ejection just a few years before it died. Smith \& Owocki (2006) have argued that these events are driven by a mechanism that appears to be independent of metallicity. Since this metallicity-independent mechanism aparently dominates the mass loss of very massive stars in the local Universe, we should not rule out the possibility that it may also be important at very low metallicity in the early Universe. If so, LBV-like blasts followed by $\mathrm{SN}$ could make similarly luminous events, and could be detected in the early Universe as well.

OK - just one more final thought. So far, the only empirical data that has been mentioned in connection with the first stars and PISNe is the abundance patterns in very very very low-metallicity stars thought to have been born in the early Universe, and there have been many illuminating talks on that subject at this meeting. Is there an analogous way to search for evidence of any signature of modern PISNe, such as in meteoritic abundance patterns or abundances of young stars or protostellar disks at the edges of very massive giant H II regions where a very massive star might have already exploded? Perhaps this may be an avenue to rule out the idea that PISNe occur locally.

\section{ACKNOWLEDGMENTS}

This contribution summarizes some of my own thoughts, but it follows directly from the research on SN 2006gy done with my collaborators: W. Li, R.J. Foley, J.C. Wheeler, D. Pooley, R. Chornock, A.V. Filippenko, J.M. Silverman, R. Quimby, J.S. Bloom, C. Hansen, and R. McCray. SN research under A.V. Filippenko at UC
Berkeley is supported by NSF grant AST-0607485 and the TABASGO Foundation, and I thank the conference organizers for partial financial support.

\section{REFERENCES}

1. Aldering, G., et al., Astrophys. J., 650, 510-527 (2006)

2. Z. Barkat, G. Rakavy, \& N. Sack, Phys. Rev. Let., 18, 379-381 (1967).

3. J.R. Bond, W.D. Arnett, \& B.J. Carr, Astrophys. J., 280, 825-847 (1984).

4. Falk, S.W., \& Arnett, W.D., Astrophys. J., Letters, 180, L65-L68 (1973).

5. Hamuy, M., et al.,Nature, 424, 651-654 (2003).

6. A. Heger \& S.E. Woosley, Astrophys. J., 567, 532-543 (2002).

7. E. Ofek et al., Astrophys. J., Letters, 659, L13-L16, (2007).

8. E. Scannapieco, P. Madau, S.E. Woosley, A. Heger, \& A. Ferrara, Astrophys. J., 633, 1031-1041 (2005).

9. N. Smith \& R. McCray, Astrophys. J., Letters, in press, (2008), astro-ph/0710.3428

10. N. Smith \& S.P. Owocki, Astrophys. J., Letters, 645, L45-L48, (2006).

11. N. Smith et al., Astrophys. J., 666, 1116-1128 (2007).

12. S.E. Woosley, S. Blinnikov, \& A. Heger, Nature, in press (2008). 